\title{
Other and Unspecified Urinary Organs ICD-O-3
}

National Cancer Institute

\section{Source}

National Cancer Institute. Other and Unspecified Urinary Organs ICD-O-3. NCI

Thesaurus. Code C148345.

Urinary org ans that are referenced in topographic category C68 of ICD-O-3. 